Pacific Journal of Mathematics

ORTHODOX SEMIGROUPS 


\title{
ORTHODOX SEMIGROUPS
}

\author{
T. E. HALL
}

An orthodox semigroup is a regular semigroup in which the idempotents form a subsemigroup. The purpose of this paper is to give structure theorems for orthodox semigroups in terms of inverse semigroups and bands.

A different structure theorem for orthodox semigroups in terms of bands and inverse semigroups has already been given by Yamada in [12]; two questions posed in [12] will be answered in the negative. The present paper is the "further paper" mentioned by the author in the final paragraph of $\S 1[5]$ and in the Acknowledgement of [5].

2. Preliminaries. We use wherever possible, and usually without comment, the notations of Clifford and Preston [2]; further, for each element $a$ in any semigroup $S$ we define $V(a)=\{x \varepsilon S: a x a=a$ and $x a x=x\}$, the set of inverses of $a$ in $S$.

RESULT 1 (from Theorem 4.6 [2]). On any band $B$ Green's relation $\mathcal{J}$ is the finest semilattice congruence and each $\mathscr{J}$-class is a rectangular band.

Let $\phi: B \rightarrow Y$ be any homomorphism of $B$ onto a semilattice $Y$ snch that $\phi \circ \phi^{-1}=\mathscr{J}$. By denoting (for all $e \in B$ ) $J_{e}$ by $E_{\alpha}$ where $e \phi=\alpha \in Y$ we obtain $B$ as a semilattice $Y$ of the rectangular bands $\left\{E_{\alpha}: \alpha \in Y\right\}$, i.e., $B=\bigcup_{\alpha \in Y} E_{\alpha}$ and for all $\alpha, \beta \in Y E_{a} \cap E_{\beta}=\square$ if $\alpha \neq \beta$, aud $E_{\alpha} E_{\beta} \subseteq E_{\alpha \beta}$. It is clear that $\{(e, \alpha) \in B \times Y: e \phi=\alpha\}$ is a subband of $B \times Y$ isomorphic to $B$.

RESULT 2 [9, Lemma 2.2]. Let $\rho$ be a congruence on a regular semigroup $S$. Then each $\rho$-class which is an idempotent of $S / \rho$ contains an idempotent of $S$.

RESUlT 3 (from Theorem 13 [7]). Let $\rho$ be any congruence contained in $\mathscr{L}$ on any semigroup $S$. Then any elements $a$ and $b$ of $S$ are $\mathscr{L}$-related in $S$ if and only if $a \rho$ and b $\rho$ are $\mathscr{L}$-related in $S / \rho$.

Henceforth we shall let $S$ denote an arbitrary orthodox semigroup.

The following result is part of [3, Theorem 3]; as noted in [4] it had previously been obtained by Schein [10]. 
RESULT 4. The relation $\mathscr{Y}=\{(x, y) \in S \times S: V(x)=V(y)\}$ is the finest inverse semigroup congruence on the orthodox semigroup $S$.

From [3, Remark 1] we see that the partition of $S$ induced by $\mathscr{Y}$ is $\{V(\mathrm{x}): x \in S\}$. Denote the band of $S$ by $B$. Then we also have from [3, Remark 1] that for any $e \in B, e \mathscr{Y}=J_{e}$ (where $J_{e}$ is the $\mathscr{J}$-class of $B$ containing $e$ ) whence, from Result 2 , the semilattice of $S / \mathscr{Y}$ is $B / \mathscr{J}$ ( $\mathscr{J}$ being Green's relation $\mathscr{J}$ on $B$ ).

For the remainder of this section $\mathscr{L}$ and $\mathscr{R}$ shall denote Green's relations $\mathscr{L}$ and $\mathscr{R}$ on $B$; as usual then $L_{x}$ and $R_{x}$ shall denote the $\mathscr{L}$-class and $\mathscr{R}$-class respectively of $B$ containg an element $x$ from $B$.

Result 5 [5, Lemma 1] or [12, Footnote 5]. For any element $a \in S$ and any element $a^{\prime} \in V(a)$,

$$
a V(a)=R_{a a^{\prime}} \text { and } V(a) a=L_{a^{\prime} a} .
$$

Result 6 [5, Lemma 2] or [12, Lemma 5]. Take any elements $a$ and $b$ in $S$.

Then

$$
a V(a)(a \mathscr{Y}) V(a) a=\{a\}
$$

whence $a=b$ if and only if the triple

$$
(a V(a), a \mathscr{Y}, V(a) a)=(b V(b), b \mathscr{Y}, V(b) b) .
$$

Henceforth, we shall identify any one element set $\{x\}$ say, with that element $x$, as is usual.

We shall now present two constructions appearing in [5]; one is of a representation of $S$ by transformations of sets and the other is of a "maximal" fundamental orthodox semigroup containing $B$ as the band of all idempotents (a semigroup $T$ is called fundamental if the only congruence contained in $\mathscr{H}$ on $T$ is the trivial congruence). This work has been generalized to regular semigroups in [6], where in fact the proofs and presentation are simpler than in [5]. For each result that we present we shall therefore refer to results in both [5] and [6].

For each element $a$ in $S$ define a transformation $\rho_{a} \in \mathscr{T}_{B / \mathscr{L}}$, the semigroup of all transformations of the set $B / \mathscr{L}$, by

$$
V(x) x \rho_{a}=V(x a) x a \text { for all } x \in B
$$

and define also a transformation $\lambda_{a}$ in $\mathscr{T}_{B / \mathscr{\Omega}}$ by $x V(x) \lambda_{a}=a x V(a x)$ for all $x \in B$.

That $\rho_{a}$ and $\lambda_{a}$ are transformations is shown in [5, Section 3] 
and also follows from [6, Remark 4]. Let $(\rho, \lambda)$ be the mapping of

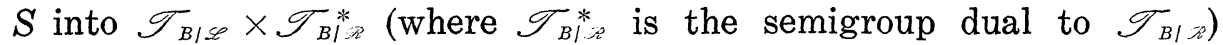
which takes each $a$ in $S$ to $\left(\rho_{a}, \lambda_{a}\right)$.

We define now an equivalence relation $\mathscr{U}$ on $B$ by $\mathscr{U}=\{(e, f) \in$ $B \times B: e B e \cong f B f\}$ and for each pair $(e, f) \in \mathscr{C}$ we let $T_{e f}$ be the set of all isomorphisms from $e B e$ onto $f B f$; for each $\alpha \in T_{e, f}$ we define further transformations $\bar{\alpha} \in \mathscr{I}_{B \mid \mathscr{E}}$ and $\overline{\bar{\alpha}} \in \mathscr{I}_{B \mid \mathcal{A}}[6$, Section 5] by

$$
L_{x} \bar{\alpha}=L_{x \alpha} \text { and } R_{x} \overline{\bar{\alpha}}=R_{x \alpha} \text { for all } x \in e B e \text {. }
$$

Further, let us consider the transformations $\rho_{e} \bar{\alpha}$ and $\lambda_{f} \overline{\overline{\alpha^{-1}}}$ (products being taken in $\mathscr{P} \mathscr{T}_{B \mid \not C}$ and $\mathscr{P} \mathscr{T}_{B \mid \not B}$ respectively) and let us put $\left(\rho_{e} \bar{\alpha}, \lambda_{f} \overline{\alpha^{-1}}\right)=\phi(\alpha)$ say. Define now

$$
W(B)=\mathbf{U}_{(e, f) \in \mathscr{r}}\left\{\left(\rho_{e} \bar{\alpha}, \lambda_{f} \overline{\overline{\alpha^{-1}}}\right): \alpha \in T_{e, f}\right\} .
$$

\section{RESULT 7.}

(i) The set $W(B)$ is a subsemigroup of $\mathscr{T}_{B \mid \mathscr{B}} \times \mathscr{T}_{B \mid: \mathscr{B}}^{*}$.

(ii) Further, $W(B)$ is a fundamental orthodox semigroup whose band of idempotents is isomorphic to $B$.

(iii) The mapping $(\rho, \lambda)$ is a homomorphism of $S$ into $W(B)$ which maps $B$ isomorphically onto the band of idempotents of $W(B)$.

(iv) The congruence $(\rho, \lambda) \circ(\rho, \lambda)^{-1}$ is the maximum congruence contained in $\mathscr{H}$ on $S$.

Result 7 can be obtained by the specialization to orthodox semigroups of the following results on regular semigroups from [6]: Lemma 4, Theorem 7 and Theorem 18 (vii). Alternatively, except for part (iii), Result 7 is contained in Theorems 1 and 5 of [5].

Result 8 [5, Theorem 2]. Take any elements $a, b \in S$. Then $a=b$ if and only if the triple

$$
\left(\lambda_{\alpha}, a \mathscr{Y}, \rho_{a}\right)=\left(\lambda_{b}, b \mathscr{Y}, \rho_{b}\right) \text {. }
$$

\section{The structure theorems.}

LEMMA 1. The mapping from $S$ into $W(B) \times(S / \mathscr{Y})$ which maps each element $a$ in $S$ to $\left(\left(\rho_{a}, \lambda_{a}\right), a \mathscr{Y}\right)$ is an isomorphism.

Proof. From Results 4 and 7 (iii) we see that the mapping is a homomorphism and from Result 8 we see that it is one-to-one.

Let now $E$ be any band and define $W(E)$ as above. Let $\left(\rho^{\prime}, \lambda^{\prime}\right)$ be the homomorphism of $E$ into $W(E)$ which corresponds to the homomorphism $(\rho, \lambda)$ of $S$ into $W(B)$ above. From Result 7 (iii) $\left(\rho^{\prime}, \lambda^{\prime}\right)$ is an isomorphism from $E$ onto the band of all idempotents of $W(E)$. 
Let us denote the band of $W(E)$ by $\bar{E}$ and for each $e \in E$ let us denote $e\left(\rho^{\prime}, \lambda^{\prime}\right)$ simply by $\bar{e}_{\text {. Let }} \mathscr{Y}_{1}$ denote the finest inverse semigroup congruence on $W(E)$, as given by Result 4 .

Now let $T$ be any inverse semigroup such that there is an idempotent-separating homomorphism $\psi$ say, from $T$ into $W(E) / \mathscr{Y}_{1}$ whose range contains all the idempotents of $W(E) / \mathscr{Y}_{1}$; if we let $Y$ denote the semilattice of $T$ then from Result $2 \psi \mid Y$ maps $Y$ isomorphically onto the semilattice of $W(E) / \mathscr{Y}_{1}$.

Let $\mathscr{Y}_{1}^{\natural}$ denote the natural homomorphism [2, Section 1.5] of $W(E)$ onto $W(E) / \mathscr{Y}_{1}$; then $x \mathscr{Y}_{1}^{\natural}=x \mathscr{Y}_{1}$ for any $x \in W(E)$.

Considering Green's relation $\mathcal{J}$ on $\bar{E}$ we have from $\S 2$ that

$$
\begin{gathered}
\left(\mathscr{Y}_{1}^{\natural} \mid \bar{E}\right) \circ\left(\mathscr{Y}_{1}^{\natural} \mid \bar{E}\right)^{-1}=\mathscr{J} \text { whence } \\
{\left[\left(\mathscr{Y}_{1}^{\natural} \mid \bar{E}\right)(\psi \mid Y)^{-1}\right] \circ\left[\left(\mathscr{Y}_{1}^{\natural} \mid \bar{E}\right)(\psi \mid Y)^{-1}\right]^{-1}=\mathscr{J}}
\end{gathered}
$$

and so we may index (Result 1 ) the $\mathscr{J}$-classes of $\bar{E}$ with the elements of $Y$ as follows: for all $\bar{e} \in \bar{E}$ if $\bar{e} \mathscr{Y}_{1}^{\sharp}(\psi \mid Y)^{-1}=\alpha \in Y$ then denote $J_{\bar{e}}$ by $\bar{E}_{\alpha}$.

Similarly, considering Green's relation $\mathscr{J}$ on $E$ and denoting $\left(\rho^{\prime}\right.$, $\left.\lambda^{\prime}\right)\left(\mathscr{Y}_{1}^{\natural} \mid \bar{E}\right)(\psi \mid Y)^{-1}$ by $\xi$ we have $\xi_{\circ} \xi^{-1}=\mathscr{J}$ whence we may index the $\mathscr{J}$-classes of $E$ with the elements of $Y$ as follows: for all $e \in E$ if $e \xi=\alpha \in Y$ then denote $J_{e}$ by $E_{\alpha}$. Clearly $e \in E_{\alpha}$ implies $\bar{e} \in \bar{E}_{\alpha}$ for all $e \in E$.

Define now $S_{1}=S_{1}(E, T, \psi)$ by

$$
S_{1}=\left\{(x, t) \in W(E) \times T: x \mathscr{V}_{1}=t \psi\right\} .
$$

\section{THEOREM 1.}

(i) The set $S_{1}=S_{1}(E, T, \psi)$ is an orthodox subsemigroup of $W(E) \times$ $T$, and conversely every orthodox semigroup is obtained in this way.

(ii) The band of $S_{1}$ is isomorphic to $E$.

(iii) The maximum inverse semigroup homomorphic image of $S_{1}$ is isomorphic to $T$.

(iv) For each element $x \in W(E)$ let $\left(x V(x), x \mathscr{Y}_{1}, V(x) x\right)$ denote $x$. Then

$$
\mathrm{S}_{1}=\left\{\left(\left(R_{\bar{e}}, t \psi, L_{\bar{f}}\right), t\right): t \in T, \bar{e} \in \bar{E}_{t t^{-1}}, \bar{f} \in \bar{E}_{t^{-1} t}\right\},
$$

where $R_{\bar{e}}$ and $L_{\bar{f}}$ are the $\mathscr{R}$-class and $\mathscr{L}$-class respectively of $\bar{E}$ containing $\bar{e}$ and $\bar{f}$ respectively.

Proof.

(i) Take any elements $(x, t),(y, u)$ in $S_{1}$. Then

$$
(x y) \mathscr{Y}_{1}^{\natural}=\left(x \mathscr{Y}_{1}^{\natural}\right)\left(y \mathscr{Y}_{1}^{\natural}\right)=(t \psi)(u \psi)=(t u) \psi
$$


whence $(x, t)(y, u)=(x y, t u) \in S_{1}$ and $S_{1}$ is a subsemigroup of $W(E) \times$ $T$. Now the set of inverses of $(x, t)$ in $W(E) \times T$ is $V(x) \times\left\{t^{-1}\right\}$ (where of course $V(x)$ denotes the set of inverses of $x$ in $W(E)$ ); take any $\left(x^{\prime}, t^{-1}\right) \in V(x) \times\left\{t^{-1}\right\}$. Then $x^{\prime} \mathscr{V}_{1}$ and $t^{-1} \psi$ are both inverses of $x \mathscr{Y}_{1}=t \psi$ in $W(E) / \mathscr{Y}_{1}$ whence $x^{\prime} \mathscr{Y}_{1}=t^{-1} \psi$ and $V(x) \times\left\{t^{-1}\right\} \subseteq S_{1}$. In particular $S_{1}$ is regular. Since $W(E) \times T$ is orthodox we now have that $S_{1}$ is orthodox.

Conversely, consider again the orthodox semigroup $S$ of $\S 2$. Let $\mathscr{Y}_{2}$ be the finest inverse semigroup congruence on $W(B)$. Then $S(\rho$, d) $\mathscr{Y}_{2}^{\natural}$ is an inverse semigroup homomorphic image of $S$ so

$$
\mathscr{Y} \subseteq\left[(\rho, \lambda) \mathscr{Y}_{2}^{\natural}\right] \circ\left[(\rho, \lambda) \mathscr{Y}_{2}^{\natural}\right]^{-1} \text {. }
$$

Let $\theta$ be the unique homomorphism from $S / \mathscr{Y}$ onto $S(\rho, \lambda) \mathscr{Y}_{2}^{\natural}$ such that $\mathscr{Y}^{\sharp} \theta=(\rho, \lambda) \mathscr{Y}_{2}^{\natural}[2$, Theorem 1.6].

The semilattices of $S / \mathscr{Y}$ and $S(\rho, \lambda) \mathscr{Y}_{2}^{\natural}$ are $B \mathscr{Y}^{\natural}$ and $B(\rho, \lambda) \mathscr{Y}_{2}^{\natural}$ respectively (Result 2), and moreover (for $\mathscr{J}$ on $B$ )

$$
\left(\mathscr{Y}^{\natural} \mid B\right) \circ\left(\mathscr{Y}^{\natural} \mid B\right)^{-1}=\mathscr{J}=\left[\left((\rho, \lambda) \mathscr{Y}_{2}^{\natural}\right) \mid B\right] \circ\left[\left((\rho, \lambda) \mathscr{Y}_{2}^{\natural}\right) \mid B\right]^{-1}
$$

so $\theta$ maps $B \mathscr{Y}^{\sharp}$ one-to-one onto $B(\rho, \lambda) \mathscr{Z}_{2}^{\natural}$. Thus $S_{1}(B, S / \mathscr{Y}, \theta)$ is defined, and further, for all $a \in S$, $\left(\left(\rho_{a}, \lambda_{a}\right), a \mathscr{Y}\right) \in S_{1}(B, S / \mathscr{Y}, \theta)$ since $(a \mathscr{Y}) \theta=a(\rho, \lambda) \mathscr{Y}_{2}^{\natural}=\left(\rho_{a}, \lambda_{a}\right) \mathscr{V}_{2}$.

Take now any element $(x, a \mathscr{Y}) \in S_{1}(B, S / \mathscr{Y}, \theta)$, where $a \in S$. Then

$$
x \mathscr{Y}_{2}=(\alpha \mathscr{Y}) \theta=a(\rho, \lambda) \mathscr{V}_{2}^{\natural}=\left(\rho_{a}, \lambda_{a}\right) \mathscr{Y}_{2}
$$

whence $V(x)=V\left(\left(\rho_{a}, \lambda_{a}\right)\right)$ in $W(B)$. Take any $a^{\prime} \in V(a)$ in $S$. Then $\left(\rho_{a^{\prime}}, \lambda_{a^{\prime}}\right) \in V(x)$ in $W(B)$ and from Result 7 (iii)

$$
\left(\rho_{a^{\prime}}, \lambda_{a^{\prime}}\right) x=\left(\rho_{e}, \lambda_{e}\right) \text { and } x\left(\rho_{a^{\prime}}, \lambda_{a^{\prime}}\right)=\left(\rho_{f}, \lambda_{f}\right)
$$

for some idempotents $e, f \in S$. Then $\left(\rho_{e}, \lambda_{e}\right) \mathscr{R}\left(\rho_{a^{\prime}}, \lambda_{a^{\prime}}\right) \mathscr{L}\left(\rho_{f}, \lambda_{f}\right)$ in $W(B)$ whence $e \mathscr{R} a^{\prime} \mathscr{L} f$ in $S$ (from Result 7 (iv), Result 3 and the result dual to Result 3). From [2, Theorem 2.18] there is an inverse $b$ say, of $a^{\prime}$ in $S$, such that $e \mathscr{L} b \mathscr{R} f$ in $S$. Thus $\left(\rho_{e}, \lambda_{e}\right) \mathscr{L}\left(\rho_{b}\right.$, $\left.\lambda_{b}\right) \mathscr{R}\left(\rho_{f}, \lambda_{f}\right)$ in $W(B)$; but also $\left(\rho_{e}, \lambda_{e}\right) \mathscr{L} x \mathscr{R}\left(\rho_{f}, \lambda_{f}\right)$ in $W(B)$ and both $x$ and $\left(\rho_{b}, \lambda_{b}\right)$ are inverses of $\left(\rho_{a^{\prime}}, \lambda_{a^{\prime}}\right)$ in $W(B)$, so from [2, Theorem 2.18] $x=\left(\rho_{b}, \lambda_{b}\right)$. Note also that $b \mathscr{Y}=a \mathscr{Y}$ (since both are inverses of $a^{\prime} \mathscr{Y}$ in $\left.S / \mathscr{Y}\right)$. Thus $(x, a \mathscr{Y})=\left(\left(\rho_{b}, \lambda_{b}\right), b \mathscr{Y}\right)$. With an observation above this gives that

$$
S_{1}(B, S / \mathscr{Y}, \theta)=\left\{\left(\left(\rho_{a}, \lambda_{a}\right), a \mathscr{Y}\right) \in W(B) \times(S / \mathscr{Y}): a \in S\right\} .
$$

From Lemma 1 we have that $S$ is isomorphic to $S_{1}(B, S / \mathscr{Y}, \theta)$.

(ii) Take any idempotent $(x, \alpha)$ say, in $S_{1}=S_{1}(E, T, \psi)$. Then $x^{2}=$ $x, \alpha^{2}=\alpha$ and $x \mathscr{Y}_{1}=\alpha \psi$ whence $x \mathscr{Y}_{1}^{a}(\psi \mid Y)^{-1}=\alpha$ and so $x \in \bar{E}_{\alpha}$. Con- 
versely, for any $\alpha \in Y$ and $x \in \bar{E}_{\alpha}$ we have $x \mathscr{Y}_{1}^{\natural}(\psi \mid Y)^{-1}=\alpha$ whence $x \mathscr{V}_{1}=\alpha \psi$ and $(x, \alpha) \in S_{1}$. Thus the band of idempotents of $S_{1}$ is $\{(x$, $\left.\alpha) \in \bar{E} \times Y: \alpha \in Y, x \in \bar{E}_{\alpha}\right\}$, which is clearly isomorphic to $\bar{E}$ (Section 2).

(iii) Let $\pi_{2}: S_{1} \rightarrow T$ be the function satisfying $(x, t) \pi_{2}=t$ for all $(x, t) \in S_{1}$, and let $\mathscr{Y}_{3}$ denote the finest inverse semigroup congruence on $S_{1}$. Then $\pi_{2}$ is a homomorphism onto $T$, an inverse semigroup, whence $\mathscr{Y}_{3} \leqq \pi_{2} \circ \pi_{2}{ }^{-1}$.

Since from the proof of (i) the set of inverses of any element ( $x$, $t)$ in $S_{1}$ is $V(x) \times\left\{t^{-1}\right\}$ we have that

$\mathscr{Y}_{3}=\left\{((x, t),(y, t)) \in S_{1} \times S_{1}: V(x)=V(y)\right.$ in $\left.W(E)\right\}$. But for any $(x, t)$, $(y, t)$ in $S_{1}$ we have $x \mathscr{Y}_{1}=t \psi=y \mathscr{Y}_{1}$ whence $V(x)=V(y)$ in $W(E)$. Thus $\pi_{2} \circ \pi_{2}^{-1} \subseteq \mathscr{Y}_{3}$, giving $\pi_{2}^{\circ} \pi_{2}^{-1}=\mathscr{V}_{3}$ and $S_{1} / \mathscr{Y}_{3}$ is isomorphic to $S_{1} \pi_{2}=T$.

(iv) We note that it is Result 6 which enables us to let $(x V(x)$, $\left.x \mathscr{Y}_{1}, V(x) x\right)$ denote $x$, for each $x \in W(E)$.

Take any element $(x, t) \in S_{1}$. Considering Green's relations $\mathscr{R}$ and $\mathscr{L}$ on $\bar{E}$ we have

$$
(x, t)=\left(\left(x V(x), x \mathscr{V}_{1}, V(x) x\right), t\right)=\left(\left(R_{x x^{\prime}}, t \psi, L_{x^{\prime} x}\right), t\right)
$$

for any $x^{\prime} \in V(x)$, from Result 5. Now $t^{-1} \psi=(t \psi)^{-1}$ and $x^{\prime} \mathscr{Y}_{1}=$ $\left(x \mathscr{Y}_{1}\right)^{-1}=(t \psi)^{-1}$ so

$$
\left(x x^{\prime}\right) \mathscr{Y}_{1}^{\natural}=\left(x \mathscr{Y}_{1}^{\natural}\right)\left(x^{\prime} \mathscr{Y}_{1}^{\natural}\right)=(t \psi)(t \psi)^{-1}=(t \psi)\left(t^{-1} \psi\right)=\left(t t^{-1}\right) \psi
$$

giving that $\left(x x^{\prime}\right) \mathscr{Y}_{1}^{\natural}(\psi \mid Y)^{-1}=t t^{-1}$ and $x x^{\prime} \in \bar{E}_{t t^{-1}}$. Similary $x^{\prime} x \in \bar{E}_{t^{-1} t}$ and so

$$
S_{1} \subseteq\left\{\left(\left(R_{\bar{e}}, t \psi, L_{\bar{f}}\right), t\right): t \in T, \bar{e} \in \bar{E}_{t t^{-1}}, \bar{f} \in \bar{E}_{t^{-1} t}\right\} .
$$

Conversely take any $t \in T$ and any $\bar{e} \in \bar{E}_{t t^{-1}}$ and $\bar{f} \in \bar{E}_{t^{-1} t}$; then $\bar{e}_{\mathcal{Y}_{1}}=$ $\left(t t^{-1}\right) \psi$ and $\bar{f} \mathscr{Y}_{1}=\left(t^{-1} t\right) \psi$. Consider $\left(\left(R_{\bar{e}}, t \psi, L_{\bar{f}}\right), t\right)$. Take any element $x \in W(E)$ such that $x \mathscr{V}_{1}=t \psi$. Then $(\bar{e} x \bar{f}) \mathscr{Y}_{1}^{\natural}=\left(\bar{e} \mathscr{Y}_{1}^{\natural}\right)\left(x \mathscr{Y}_{1}^{\natural}\right)\left(\bar{f} \mathscr{Y}_{1}^{\natural}\right)=$ $\left[\left(t t^{-1}\right) \psi\right](t \psi)\left[\left(t^{-1} t\right) \psi\right]=t \psi$. Take any $x^{\prime} \in V(x)$ and put $\bar{e} x \bar{f}=y$ and $\bar{f} x^{\prime} \bar{e}=y^{\prime}$. Then $y^{\prime} \in V(y)\left[10\right.$, Theorem 1.10], whence $y^{\prime} \mathscr{Y}=(t \psi)^{-1}=$ $t^{-1} \psi$. Thus $\left(y y^{\prime}\right) \mathscr{Y}_{1}=\left(t t^{-1}\right) \psi$ giving $y y^{\prime} \in \bar{E}_{t t^{-1}}$ and similarly $y^{\prime} y \in \bar{E}_{t^{-1} t}$. Now $\bar{e}, y y^{\prime} \in \bar{E}_{t t-1}$, a rectangular band, so

$$
y y^{\prime}=(\bar{e} x \bar{f})\left(\bar{f} x^{\prime} \bar{e}\right)=\bar{e} y y^{\prime} \bar{e}=\bar{e}
$$

and similarly $y^{\prime} y=\bar{f}$. Thus

$$
\left(\left(R_{\bar{e}}, t \psi, L_{\bar{f}}\right), t\right)=\left(\left(y V(y), y \mathscr{Y}_{1}, V(y) y\right), t\right)=(y, t) \in S_{1} .
$$

Therefore

$$
S_{1}=\left\{\left(\left(R_{\bar{e}}, t \psi, L_{\bar{f}}\right), t\right): t \in T, \bar{e} \in \bar{E}_{t t^{-1}}, \bar{f} \in \bar{E}_{t_{-}{ }^{1} t}\right\} .
$$


REMARK 1. Let $Z$ denote the semilattice of $S / \mathscr{Y}$ and index the $\mathcal{J}$-classes of $B$ with the elements of $Z$ in the natural way. For each element $a \in S$ let ( $a V(a), a \mathscr{Y}, V(a) a)$ denote $a$ and consider the $\mathscr{R}$ and $\mathscr{L}$-classes of $B$. Then the method used to prove (iv) also gives that

$$
\begin{aligned}
S= & \left\{\left(R_{e}, v, L_{f}\right) \in(B / \mathscr{R}) \times(S / \mathscr{Y}) \times(B / \mathscr{L}): v \in S / \mathscr{Y}, e \in E_{v v-1}\right. \\
& \text { and } \left.f \in E_{v-\mathbf{1}_{v}}\right\} .
\end{aligned}
$$

CoRollary 1 (to the proof). Consider the arbitrary band $E$ and any inverse semigroup $U$. Then there exists an orthodox semigroup whose band is $E$ and whose maximum inverse semigroup image is isomorphic to $U$ if and only if there is a homomorphism from $U$ into $W(E) / \mathscr{Y}_{1}$ which maps the idempotents of $U$ one-to-one onto the idempotents of $W(E) / \mathscr{Y}_{1}$.

Let us now define a subset $S_{2}=S_{2}(E, T, \psi)$ of $(E / \mathscr{R}) \times T \times$ $(E / \mathscr{L})$ by

$$
S_{2}=\left\{\left(R_{e}, t, L_{f}\right): t \in T, e \in E_{t t^{-1}} \text { and } f \in E_{t-1_{t}}\right\} \text {. }
$$

Take any element $\left(R_{e}, t, L_{f}\right)$ in $S_{2}$. Then $\bar{e} \in \bar{E}_{t t^{-1}}$ and $\bar{f} \in \bar{E}_{t^{-1} t}$ whence $\left(\left(R_{\bar{e}}, t \psi, L_{\bar{f}}\right), t\right) \in S_{1}$, where $R_{\bar{e}}$ and $L_{\bar{f}}$ are the $\mathscr{R}$-class and $\mathscr{L}$-class respectively of $\bar{E}$ containing $\bar{e}$ and $\bar{f}$ respectively. Clearly now we may define a mapping $\Psi$ of $S_{2}$ into $S_{1}$ by

$$
\left(R_{e}, t, L_{f}\right) \Psi=\left(\left(R_{\bar{e}}, t \psi, L_{\bar{f}}\right), t\right)
$$

for any element $\left(R_{e}, t, L_{f}\right) \in S_{2}$. It is also clear that $\Psi$ is one-to-one and it is routine to show that $\Psi$ is onto $S_{1}$. Thus $\Psi$ is a one-to-one correspondence between $S_{2}$ and $S_{1}$.

Let us denote by juxtaposition the unique multiplication on $S_{2}$ which makes $\Psi$ an isomorphism from $S_{2}$ onto $S_{1}$; then for any elements $\left(R_{e}, t, L_{f}\right)$ and $\left(R_{g}, u, L_{h}\right)$ in $S_{2}$

$$
\left(R_{e}, t, L_{f}\right)\left(R_{g}, u, L_{h}\right)=\left[\left(R_{e}, t, L_{f}\right) \Psi\left(R_{g}, u, L_{h}\right) \Psi\right] \Psi^{-1} .
$$

From Result 6 and Theorem 1 (iv) $\left(\left(R_{\bar{e}}, t \psi, L_{\bar{f}}\right), t\right)$ denotes the element $\left(R_{\bar{e}}(t \psi) L_{\bar{f}}, t\right)$ of $S_{1}$; thus

$$
\left(R_{e}, t, L_{f}\right) \Psi=\left(R_{\bar{e}}(t \psi) L_{\bar{f}}, t\right)
$$

for any element $\left(R_{e}, t, L_{f}\right)$ in $S_{2}$.

For each idempotent $x \in W(E)$ let $\widetilde{x}$ denote $x\left(\rho^{\prime}, \lambda^{\prime}\right)^{-1}$; then $\overline{\widetilde{x}}=x$ for all $x \in \bar{E}$ and $\widetilde{e}=e$ for all $e \in E$. Then for any elements $\left(R_{e}, t, L_{f}\right)$ and $\left(R_{g}, u, L_{h}\right)$ in $S_{2}$ 


$$
\left(R_{e}, t, L_{f}\right)\left(R_{g}, u, L_{h}\right)=\left(R_{\widetilde{z} z^{\prime}}, t u, L_{z^{\prime} z}\right)
$$

where (in $W(E)) R_{\bar{e}}(u \psi) L_{\bar{f}}=x, R_{\bar{g}}(u \psi) L_{\bar{h}}=y, x y=z$ and $z^{\prime} \in V(z)$; this is because $(t u) \psi=(x y) \mathscr{V}_{1}=z \mathscr{Y}_{1}$ and

$$
\left(R_{\tilde{z} z^{\prime}}, t u, L_{\tilde{z}^{\prime} z}\right) \Psi=\left(\left(R_{z z^{\prime}},(t u) \psi, L_{z^{\prime} z}\right), t u\right)=(z, t u)=(x y, t u) .
$$

We restate these facts in the next theorem.

THEOREM 2. Let $S_{2}=S_{2}(E, T, \psi)$ be the subset of $(E / \mathscr{R}) \times T \times$ $(E / \mathscr{L})$ given by

$S_{2}=\left\{\left(R_{e}, t, L_{f}\right): t \in T, e \in E_{t t^{-1}}\right.$ and $\left.f \in E_{t^{-1} t}\right\}$ and let a multiplication on $S_{2}$ be given by (for any elements $\left(R_{e}, t, L_{f}\right)$ and $\left(R_{g}, u, L_{h}\right)$ in $\left.S_{2}\right)$

$$
\left(R_{e}, t, L_{f}\right)\left(R_{g}, u, L_{h}\right)=\left(R_{\tilde{z}^{\prime}}, t u, L_{z^{\prime} z}\right)
$$

where (for the $\mathscr{R}$ and $\mathscr{L}$-classes of $\bar{E}$ we have) $R_{\bar{e}}(t \psi) L_{\bar{f}}=x, R_{\bar{g}}(u \psi) L_{\bar{h}}=$ $y, x y=z$ and $z^{\prime} \in V(x)$ (all in $W(E)$ ). Then $S_{2}(E, T, \psi)$ is a semigroup isomorphic to $S_{1}(E, T, \psi)$.

4. Some counter-examples.

4.1. Let $T$ denote the bicyclic semigroup [2, Section 1.12]. We shall construct a band $B$ which is an $\omega$-chain of rectangular bands and such that there is no orthodox semigroup $S$ with band $B$ and with $T$ as a homomorphic image.

Let $Y$ be the semilattice of $T$; then $Y$ is an $\omega$-chain. For each $\alpha \in Y$ let $E_{\alpha}$ be a rectangular band such that, for all $\alpha, \beta \in Y$, if $\alpha \neq$ $\beta$ then $E_{\alpha} \cap E_{\beta}=\square$ and $\left|E_{\alpha}\right| \neq\left|E_{\beta}\right|$. Put $B=\bigcup_{\alpha \in Y} E_{\alpha}$ and, following Clifford [1] extend the multiplications of the bands $\left\{E_{\alpha}: \alpha \in Y\right\}$ to a multiplication for $B$ as follows: for any $e, f \in B$, where $e \in E_{\alpha}$ and $f \in E_{\beta}$ say, define

$$
e f=\left\{\begin{array}{l}
e \text { if } \alpha<\beta \\
e f \text { as in } E_{\alpha} \text { if } \alpha=\beta \\
f \text { if } \alpha>\beta .
\end{array}\right.
$$

Note that if $\alpha>\beta$ then $e f=f e=f$. It is routine to show that this multiplication is associative (alternatively see [8]) and that then the band $B$ is an $\omega$-chain $Y$ of the rectangular bands $\left\{E_{\alpha}: \alpha \in Y\right\}$. Also, if $e \in E_{\alpha}$ and $f \in E_{\beta}(\alpha, \beta \in Y)$ then $e B e=\{e\} \cup\left(\bigcup_{r<\alpha} E_{\gamma}\right)$ whence $e B e$ is isomorphic to $f B f$ if and only if $\alpha=\beta$. From [5, Main Theorem] any orthodox semigroup, $S$ say, with band $B$ is a union of groups. But any homomorphic image of a semigroup which is a union of groups is also a union of groups; thus $T$ is not the maximum inverse semigroup homomorphic image of $S$. 
REMARK 2. The band $B$ just defined is one of a class of bands called, by the author, almost commutative bands; a band $E$ is called almost commutative if, for any $e, f \in E, J_{e} \neq J_{f}$ implies $e f=f e$. It is easily shown (See [8])) that a band $E$ is almost commutative if and only if, for $e, f \in E, J_{e}>J_{f}$ implies $e>f$ (where $J_{e}>J_{f}$ means that $E^{1} e E^{1} \supset E^{1} f E^{1}[2$, Section 2.1] and $e>f$ means that $e f=f e=f \neq$ $e[2$, Section 1.8]). A determination of the structure of almost commutative bands in terms of semilattices is given in [8].

REMARK 3. The band $B$ and inverse semigroup $T$ above answer in the negative the first question posed on page 269 [12]. We now briefly give alternative examples of a different nature. Let $E$ consist of the matrices

$$
\left(\begin{array}{lll}
0 & 0 & 0 \\
0 & 0 & 0 \\
0 & 0 & 0
\end{array}\right),\left(\begin{array}{lll}
1 & 0 & 0 \\
0 & 0 & 0 \\
0 & 0 & 0
\end{array}\right),\left(\begin{array}{lll}
0 & 0 & 0 \\
0 & 1 & 0 \\
0 & 0 & 0
\end{array}\right),\left(\begin{array}{lll}
0 & 0 & 0 \\
0 & 1 & 1 \\
0 & 0 & 0
\end{array}\right),\left(\begin{array}{lll}
1 & 0 & 0 \\
0 & 1 & 0 \\
0 & 0 & 1
\end{array}\right)
$$

and let $T_{1}$ consist of the matrices

$$
\left(\begin{array}{ll}
0 & 0 \\
0 & 0
\end{array}\right),\left(\begin{array}{ll}
1 & 0 \\
0 & 0
\end{array}\right),\left(\begin{array}{ll}
0 & 1 \\
0 & 0
\end{array}\right),\left(\begin{array}{ll}
0 & 0 \\
1 & 0
\end{array}\right),\left(\begin{array}{ll}
0 & 0 \\
0 & 1
\end{array}\right),\left(\begin{array}{ll}
1 & 0 \\
0 & 1
\end{array}\right),\left(\begin{array}{ll}
0 & 1 \\
1 & 0
\end{array}\right) .
$$

Under matrix multiplication $E$ is a band, $T_{1}$ is an inverse semigroup with semilattice isomorphic to $E / \mathscr{J}$, and there is no orthodox semigroup $S$ say, with band $E$ and such that $S / \mathscr{Y}$ is isomorphic to $T_{1}$.

4.2. We now give two non-isomorphic orthodox semigroups $S_{1}$ and $S_{2}$ whose bands are isomorphic and whose maximum inverse semigroup homomorphic images are isomorphic. This answers the second question on page 269 [12] in the negative. The referee has pointed out that this question has also been essentially answered in the last remark of Yamada [13].

Let $S_{1}$ consist of the matrices

$$
\left(\begin{array}{ll}
1 & 0 \\
0 & 0
\end{array}\right),\left(\begin{array}{ll}
1 & 0 \\
1 & 0
\end{array}\right),\left(\begin{array}{ll}
1 & 0 \\
0 & 1
\end{array}\right),\left(\begin{array}{rr}
1 & 0 \\
1 & -1
\end{array}\right)
$$

and let $S_{2}$ consist of the matrices

$$
\left(\begin{array}{lll}
1 & 0 & 0 \\
0 & 0 & 0 \\
0 & 0 & 0
\end{array}\right),\left(\begin{array}{lll}
1 & 0 & 0 \\
1 & 0 & 0 \\
0 & 0 & 0
\end{array}\right),\left(\begin{array}{lll}
1 & 0 & 0 \\
0 & 1 & 0 \\
0 & 0 & 1
\end{array}\right),\left(\begin{array}{rrr}
1 & 0 & 0 \\
0 & 1 & 0 \\
0 & 0 & -1
\end{array}\right) .
$$


Under matrix multiplication $S_{1}$ and $S_{2}$ are orthodox semigroups.

The bands of $S_{1}$ and $S_{2}$ are both two-element left zero semigroups with an identity adjoined and the maximum inverse semigroup homomorphic images are both two-element groups with a zero adjoined. But $\mathscr{H}$ is a congruence on $S_{2}$ and not on $S_{1}$, so $S_{1}$ and $S_{2}$ are not isomorphic.

\section{REFERENCES}

1. A. H. Clifford, Naturally totally ordered commutative semigroups, Amer. J. Math., 76 (1954), 631-646.

2. A. H. Clifford and G. B. Preston, The algebraic theory of semigroups, Vols. I and II, Amer. Math. Soc., Providence, R. I., 1961 and 1967.

3. T. E. Hall, On regular semigroups whose idempotents form a subsemigroup, Bull. Austral. Math. Soc., 1 (1969), 195-208.

4. - On regular semigroups whose idempotents form a subsemigroup: Addenda, Bull. Austral. Math. Soc., 3 (1970), 287-288.

5. - On orthodox semigroups and uniform and antiuniform bands, J. Algebra, 16 (1970), 204-217.

6. - On regular semigroups, J. Algebra, (to appear).

7. - Congruences and Green's relations on regular semigroups, Glasgow Math.

J., (to appear).

8. — Almost commutative bands, Glasgow Math. J., (to appear).

9. G. Lallement, Congruences et équivalences de Green sur un demi-groupe régulier,

C. R. Acad. Sci. Paris, Série A, 262 (1966), 613-616.

10. B. M. Schein, On the theory of generalized groups and generalized heaps, (Russian), Theory of semigroups and appl. I (Russian), 286-324, (Izdat. Saratov. Univ., Saratov, 1965).

11. M. Yamada, Regular semigroups whose idempotents satisfy permutation identities, Pacific J. Math., 21 (1967), 371-392.

12. - On a regular semigroup in which the idempotents form a band, Pacific J. Math., 33 (1970), 261-272.

13. Construction of inversive semigroups, Mem. Fac. Lit. \& Sci., Shimane Univ., Nat. Sci., 4 (1971), 1-9.

Received February 12, 1971 and in revised form August 2, 1971. This research was supported by a Nuffield Travelling Fellowship. The author thanks the referee for references [1] and [13] and for suggesting several improvements.

UNIVERSITY OF STIRLING

SCOTLAND 


\title{
PACIFIC JOURNAL OF MATHEMATICS
}

\author{
EDITORS
}

\author{
H. SAMELSON \\ Stanford University \\ Stanford, California 94305 \\ C. R. HOBBY \\ University of Washington \\ Seattle, Washington 98105
}

J. DugundJI

Department of Mathematics

University of Southern California

Los Angeles, California 90007

RICHARD ARENS

University of California

Los Angeles, California 90024

\section{ASSOCIATE EDITORS}
E. F. BeCKenBaCH
B. H. NeUMANN
F. WOLF
K. Yoshida

\section{SUPPORTING INSTITUTIONS}

\author{
UNIVERSITY OF BRITISH COLUMBIA \\ CALIFORNIA INSTITUTE OF TECHNOLOGY \\ UNIVERSITY OF CALIFORNIA \\ MONTANA STATE UNIVERSITY \\ UNIVERSITY OF NEVADA \\ NEW MEXICO STATE UNIVERSITY \\ OREGON STATE UNIVERSITY \\ UNIVERSITY OF OREGON \\ OSAKA UNIVERSITY
}

\author{
UNIVERSITY OF SOUTHERN CALIFORNIA \\ STANFORD UNIVERSITY \\ UNIVERSITY OF TOKYO \\ UNIVERSITY OF UTAH \\ WASHINGTON STATE UNIVERSITY \\ UNIVERSITY OF WASHINGTON \\ AMERICAN MATHEMATICAL SOCIETY \\ NAVAL WEAPONS CENTER
}

The Supporting Institutions listed above contribute to the cost of publication of this Journal, but they are not owners or publishers and have no responsibility for its content or policies.

Mathematical papers intended for publication in the Pacific Journal of Mathematics should be in typed form or offset-reproduced, (not dittoed), double spaced with large margins. Underline Greek letters in red, German in green, and script in blue. The first paragraph or two must be capable of being used separately as a synopsis of the entire paper. The editorial "we" must not be used in the synopsis, and items of the bibliography should not be cited there unless absolutely necessary, in which case they must be identified by author and Journal, rather than by item number. Manuscripts, in duplicate if possible, may be sent to any one of the four editors. Please classify according to the scheme of Math. Rev. Index to Vol. 39. All other communications to the editors should be addressed to the managing editor, Richard Arens, University of California, Los Angeles, California, 90024.

50 reprints are provided free for each article; additional copies may be obtained at cost in multiples of 50 .

The Pacific Journal of Mathematics is published monthly. Effective with Volume 16 the price per volume (3 numbers) is $\$ 8.00$; single issues, $\$ 3.00$. Special price for current issues to individual faculty members of supporting institutions and to individual members of the American Mathematical Society: $\$ 4.00$ per volume; single issues $\$ 1.50$. Back numbers are available.

Subscriptions, orders for back numbers, and changes of address should be sent to Pacific Journal of Mathematics, 103 Highland Boulevard, Berkeley, California, 94708.

PUBLISHED BY PACIFIC JOURNAL OF MATHEMATICS, A NON-PROFIT CORPORATION

Printed at Kokusai Bunken Insatsusha (International Academic Printing Co., Ltd.), 270, 3chome Totsuka-cho, Shinjuku-ku, Tokyo 160, Japan. 


\section{Pacific Journal of Mathematics}

\section{Vol. 39, No. $3 \quad$ July, 1971}

William O'Bannon Alltop, 5-designs in affine spaces ................... 547

B. G. Basmaji, Real-valued characters of metacyclic groups ................. 553

Miroslav Benda, On saturated reduced products....................... 557

J. T. Borrego, Haskell Cohen and Esmond Ernest Devun, Uniquely representable semigroups. II.......................................

George Lee Cain Jr. and Mohammed Zuhair Zaki Nashed, Fixed points and stability for a sum of two operators in locally convex spaces ....................

Donald Richard Chalice, Restrictions of Banach function spaces ...............

Eugene Frank Cornelius, Jr., A generalization of separable groups ..............

Joel L. Cunningham, Primes in products of rings ......................

Robert Alan Morris, On the Brauer group of $Z$.

593

603

615

David Earl Dobbs, Amitsur cohomology of algebraic number rings ...............

Charles F. Dunkl and Donald Edward Ramirez, Fourier-Stieltjes transforms and

weakly almost periodic functionals for compact groups ...................

Hicham Fakhoury, Structures uniformes faibles sur une classe de cônes et

d'ensembles convexes ......................................

Leslie R. Fletcher, A note on C $\theta \theta$-groups.

Humphrey Sek-Ching Fong and Louis Sucheston, On the ratio ergodic theorem for

semi-groups............................................

James Arthur Gerhard, Subdirectly irreducible idempotent semigroups ...........

Thomas Eric Hall, Orthodox semigroups.....................

Marcel Herzog, $C \theta \theta$-groups involving no Suzuki groups ..........

669

687

John Walter Hinrichsen, Concerning web-like continua ..........

691

Frank Norris Huggins, A generalization of a theorem of F. Riesz.

695

Carlos Johnson, Jr., On certain poset and semilattice homomorphisms

703

Alan Leslie Lambert, Strictly cyclic operator algebras ...........

717

Howard Wilson Lambert, Planar surfaces in knot manifolds . . .

727

Robert Allen McCoy, Groups of homeomorphisms of normed linear spaces ....... 735

T. S. Nanjundiah, Refinements of Wallis's estimate and their generalizations ...... 745

Roger David Nussbaum, A geometric approach to the fixed point index .......... 751

John Emanuel de Pillis, Convexity properties of a generalized numerical range .... 767

Donald C. Ramsey, Generating monomials for finite semigroups ....

783

William T. Reid, A disconjugacy criterion for higher order linear vector differential equations...

Roger Allen Wiegand, Modules over universal regular rings...

Kung-Wei Yang, Compact functors in categories of non-archimedean Banach

spaces.

R. Grant Woods, Correction to: "Co-absolutes of remainders of Stone-Čech compactifications".

Ronald Owen Fulp, Correction to: "Tensor and torsion products of

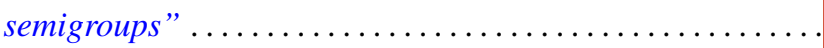

Bruce Alan Barnes, Correction to: "Banach algebras which are ideals in a banach 